\title{
The Value of Companion Diagnostics: Overcoming Access Barriers to Transform Personalised Health Care into an Affordable Reality in Europe
}

\author{
Victoria Wurcel $^{\mathrm{a}}$ Olivier Perche ${ }^{c}$ Daniel Lesteven ${ }^{d}$ Doris-Ann Williams $^{f}$ \\ Birgit Schäfer $^{\text {h }}$ Colin Hopley ${ }^{g}$ Rebecca Jungwirth ${ }^{j} \quad$ Anne Postulka $^{\text {e }}$ \\ Raf Pasmans $^{b}$ Lisse-Lotte Hermansson ${ }^{\mathrm{i}}$ Markus Ott ${ }^{\mathrm{k}}$ Valeria Glorioso' \\ aEDMA, The European Diagnostic Manufacturers Association, Member of MedTech Europe, Brussels, and ${ }^{\mathrm{b} B i o c a r t i s,}$ \\ Mechelen, Belgium; ' ${ }^{\circ}$ oche Diagnostics/SIDIV, and d Janssen Diagnostics/SIDIV, The French In Vitro Diagnostics \\ Association, Paris, and ${ }^{e}$ Cepheid, Maurens-Scopont, France; ${ }^{\mathrm{f}} \mathrm{BIVDA}$, The British In Vitro Diagnostics Association, \\ London, and ${ }^{9} \mathrm{BD}$, Oxford, UK; hVDGH, The German Association of Diagnostic Manufacturers, Berlin, Germany;

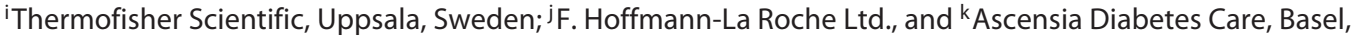 \\ Switzerland; 'Assobiomedica, Milan, Italy
}

\section{Key Words}

Companion diagnostics - Diagnostic tests - Equitable access · In vitro diagnostics · Personalised health care Personalised medicine $\cdot$ Reimbursement challenges - Sustainable health systems $\cdot$ Value of diagnostic information

\begin{abstract}
Personalised health care is an evolution, moving away from a disease-focused model of care, translating scientific and technological advances into benefits for patients, and placing them at the centre of the patients' health and care. Companion diagnostics emerge as a very specific and special group of in vitro diagnostics among the different technologies shaping the personalised health care spectrum. Companion diagnostics provide highly valuable information, allowing patients, health practitioners and payers to decide with a higher level of certainty on the potential benefits of a treatment or care pathway. Decreasing uncertainty may result in a more
\end{abstract}

\section{KARGER}

E-Mail karger@karger.com www.karger.com/phg

\section{C) 2016 The Author(s) \\ Published by S. Karger AG, Basel $1662-4246 / 16 / 0193-0137 \$ 39.50 / 0$}

This article is licensed under the Creative Commons AttributionNonCommercial-NoDerivatives 4.0 International License (CC BY NC-ND) (http://www.karger.com/Services/OpenAccessLicense) Usage and distribution for commercial purposes as well as any distribution of modified material requires written permission. efficient selection of treatments and care, targeted at subpopulations that are most likely to benefit. Companion diagnostics account for a minimal portion of the already small expenditure on in vitro diagnostics (far less than $1 \%$ of total health care expenditure), and yet they provide the means to limit inefficient use of health care resources while optimising patient outcomes. It is clear that equal access to personalised health care is still an issue across the EU. One of the most common perceived barriers is affordability. The investment in companion diagnostics can provide long-term value for patients and health care systems, shifting resources to areas of need. Health systems do not fully recognise yet the value that companion diagnostics bring to make personalised health care more affordable across the EU. This inhibits patient access to personalised treatments and care, preventing improved outcomes. In many countries, market access frame-

O.P., D.L. R.J., A.P., R.P. and L.-L.H. are members of the EDMA HTA task force companion diagnostics group.
Dr. Victoria Wurcel

European Diagnostic Manufacturers Association Member of MedTech Europe, Rue Joseph II 40 BE-1000 Brussels (Belgium)

E-Mail v.wurcel@medtecheurope.org 
works for diagnostic tests are fragmented and not aligned with specific funding and reimbursement mechanisms, discouraging the use of these tests. Emerging evidence shows that patients are missing out on the appropriate tests and treatments while a reduction in the inefficient use of health care resources is not realised. This article outlines some of these market access barriers for companion diagnostics in the $\mathrm{EU}$, including reimbursement challenges specific to some member states (Germany, the UK, and France). Furthermore, proposals addressing barriers and increasing timely patient access to companion diagnostics in the EU are presented.

(c) 2016 The Author(s)

Published by S. Karger AG, Basel

\section{Introduction}

Major changes are taking place in the way health care is conceived, applied and paid for. Health care providers and decision makers are starting to prioritise management strategies based on their potential to improve results for patients (health outcomes, quality of health care), ensuring that health care resources are wisely spent and will sustain for future generations.

These intelligent management strategies refer not only to treatments (medicines and medical devices), but also to in vitro diagnostics including tests to predict evolution of disease and treatment response, among others. The twin objectives of advancing health and ensuring sustainable use of resources are often complementary and comprise the basis of the value of health care and health care interventions [1].

Additional changes occur as a consequence of the dramatic scientific advances in our knowledge of the human genome [2]. We now realise that people develop disease and respond to treatments differently based on their genetic make-up, which is slightly different from everyone else's. Traditional 'one-size-fits-all' approaches to health care, based on broad population average response, can lead to unnecessary adverse events, delay most appropriate patient management strategies and stimulate the use of extra health care resources.

In this context, personalised health care can be a way forward, translating scientific and technological advances into benefits targeted to the specific needs of each patient.

Among the different technologies shaping this personalised health care spectrum, companion diagnostics emerge as a very special group of in vitro diagnostics that will be the focus of this article.

Companion diagnostics are a very specific group of in vitro diagnostic tests providing information which influ- ences the benefit-risk ratio of a specific treatment. Their diagnostic information helps to segment patients according to the presence or absence of a specific characteristic (in the genetic material or in non-genetic markers) that will determine the individual patient's response to a targeted therapy.

Enabling patients, health practitioners and payers to decide with a higher level of certainty on the potential benefits of a treatment and care pathway, companion diagnostics facilitate a more efficient selection of treatments, targeted at subpopulations that are most likely to benefit.

Companion diagnostics account for a minimal portion of the already small expenditure on in vitro diagnostics - far less than $1 \%$ of the total health care expenditure - and yet they can reduce the inefficient use of health care resources, while optimising patient outcomes.

Since the value of companion diagnostics as enablers of better patient outcomes, while facilitating an affordable selection of medicines and the efficient use of resources, is not yet fully recognised by EU health systems, equal access to affordable personalised health care remains an issue across the EU.

In many countries, market access frameworks for diagnostic tests are fragmented and not aligned with specific funding and reimbursement mechanisms, providing a disincentive to use these tests. There is an emerging body of evidence that patients are missing out on the appropriate tests and treatments while a reduction in the inefficient use of health care resources is not realised.

This article outlines some of those market access barriers for companion diagnostics in the EU, including reimbursement challenges specific to some member states (Germany, the UK, and France). Furthermore, proposals addressing barriers and increasing timely patient access to companion diagnostics in the $\mathrm{EU}$ are presented.

\section{Challenges and Recommendations to Improve Reimbursement and Patient Access to Companion Diagnostic Tests in Germany}

\section{Challenges}

In Germany's ambulatory sector, the practical definition and execution of the reimbursement processes for companion diagnostics is driven by the medical self-administration. The main organisation included is Gemeinsamer Bundesausschuss (G-BA), consisting of representatives from all relevant stakeholders in the public health 
care sector (physicians, hospitals, statutory health insurance companies and patients). The federal authority (Health Ministry) does not play an active role, its influence being limited to the set-up of legal rules for reimbursement processes.

From a patient access perspective, innovative medicines requiring a companion diagnostic have to be reimbursed on the day of their approval, following the socalled 'AMNOG process' [3]. Within 3 months of market authorisation, the Federal Joint Committee needs to provide a decision on the additional benefit of the medicine over the appropriate comparator. For this purpose, the manufacturer submits a medical dossier based on the authorisation documents, summarising all studies that have been performed to support that claim.

On the other hand, innovative companion diagnostic tests have to undergo a very lengthy and complex process, interacting with different stakeholders, in order to achieve reimbursement. The committee in charge is the 'Bewertungsausschuss' (Evaluation Committee), requiring different documents and evidence to prove the claimed benefit of the test.

A key challenge for inclusion of companion diagnostics in the fee schedule of the outpatient sector in the German public health system stems from the lack of clear established timelines for the reimbursement processes. As a result, those processes are not suited for this kind of tests and result in reimbursement delays impeding effective patient access to companion diagnostics and corresponding medicine.

The example of adjuvant chemotherapy for breast cancer, where doctors and patients face the situation that an upstream diagnostic test is required, but no reimbursement exists for this companion diagnostic test highlights this situation.

A new law enforced in July 2015, setting up time limits for the evaluation procedure of innovative diagnostic tests, might be a first step in the right direction to address some of these issues [4].

The situation is not as difficult in the in-patient sector, as hospital reimbursement in Germany is based on a disease-related group system and more clear processes and timelines are in place.

\section{Recommendation}

Due to the special nature of companion diagnostic tests, clear, appropriate and timely patient access processes are urgently needed in the German ambulatory sector.

Value of Companion Diagnostics

\section{Challenges and Recommendations to Improve Provision and Equitable Patient Access to Molecular Cancer Diagnostics in England}

\section{Challenges}

Molecular cancer diagnostic tests such as companion diagnostics have been available in the NHS in England for some time and are recognised as having the potential to improve patient outcomes and produce significant benefits for the service [5-7]. Despite a consensus on their benefits, access to molecular testing is variable in England and there are a number of systemic barriers within the NHS that inhibit their uptake. In particular, a fragmented commissioning structure and lack of associated funding for tests can be highlighted $[5,8]$.

The demand for molecular testing is growing [5] as new targeted medicines with new associated biomarkers, relevant for different patient subpopulations, become available. However, a gap is emerging in England where the provision of testing for a biomarker does not increase sufficiently to meet this demand [5]. An analysis of molecular diagnostic provision done by Cancer Research UK found that, although there was an increase in the volume of molecular cancer diagnostic tests carried out through the NHS between 2011 and 2014, there is still a significant level of unmet demand [5]. In 2014, the expected number of tests for the population was 59,294, but the provision of testing was only 34,675 , i.e. a difference of around 24,000 tests [5]. This included 16,000 patients with non-small cell lung cancer who would have been eligible for testing. Of these patients, 3,500 would have been eligible for targeted medicines that could have prolonged their progression-free survival, but missed out [5].

The key reason for the gap between the provision and demand for molecular diagnostic testing is a lack of specific funding for the tests. There is currently no explicit fee per test funding for molecular diagnostics in England, so commissioning is achieved through several channels $[5,9]$ :

- funding the test as part of the bundle of care within the agreed tariff for the procedure;

- on a test-by-test basis;

- through a block contract agreement with specialist treatment centres.

A common feature of these funding mechanisms, contributing to inconsistent use, is that the budget for tests often comes from a different silo to that of the treatment. This can limit the willingness to pay by the test budget holder, as it is seen solely as an additional cost [9]. Fur-

Public Health Genomics 2016;19:137-143 
thermore, ad hoc funding arrangements made with pharmaceutical companies have created some inconsistency and their existence has lowered the incentive for the NHS to address this issue [5]. These arrangements are currently being withdrawn by manufacturers, exacerbating the problem of variable access to molecular tests.

Other systemic barriers include [9]:

- confusion about the extent to which a molecular test can be funded by the cancer drug fund and uncertainty about its future;

- overlap with the 100,000 Genome Project.

\section{Recommendations}

Starting in 2011, a number of commitments have been made to develop a specific commissioning structure for molecular diagnostics. These include the Department of Health's 2011 Cancer Strategy that pledged to 'develop a commissioning and funding structure to enable the efficient delivery of high quality molecular diagnostic testing through centres of excellence' [8]. In December 2012, the Department of Health's Cancer Policy Team published proposals for ensuring equitable access to complex molecular diagnostic testing for cancer patients [8]. Progress in implementing these commitments has been slow and access to these tests remains fragmented and variable [10].

Cancer Research UK has called for commissioning of cancer molecular diagnostics and, in 2015, the Independent Cancer Taskforce published its cancer strategy for England, making almost 100 recommendations, such as: NHS England should transform access to molecular diagnostics to guide treatment for cancer by $[9,11]$ :

- specific commissioning to unlock funding and provide access to molecular diagnostic tests in 2016, starting with melanoma, lung, colorectal, breast and all paediatric cancers, before broadening out to all cancer types with treatments subject to molecular tests;

- adding the use of molecular diagnostic tests to the Cancer Outcomes and Services Dataset (COSD);

- undertaking an annual review on molecular diagnostics capacity.

These recommendations are now being considered by NHS England as part of their next spending review negotiations.

The British IVD Industry Association (BIVDA) has put forward the following recommendations:

- molecular diagnostics should be embedded at every stage of the cancer management pathway, from assessing which is the best type of treatment to monitoring its effectiveness $[9,12]$;
- BIVDA also calls for commissioning and policy decisions relating to the provision and access to molecular diagnostics, ensuring equal and timely patient access to these diagnostic tests;

- as new tests are rapidly being developed, any future systems should be flexible to support early clinical leadership and adoption;

- arrangements should be put in place to make additional tests available as and when new evidence emerges and to decommission tests if and when they are superseded.

Molecular cancer diagnostic tests are relatively affordable, costing much less than the treatment themselves. Considering that NHS expenditure on cancer has increased from GBP 3.19 billion in 2003/4 to GBP 5.68 billion in 2012/13 [13] and the full provision of lung, colorectal, and melanoma molecular diagnostics costs GBP 13.32 million [5], funding these tests can represent a strategic investment by the health service, improving patient outcomes and experiences while reducing the cost of treatment and care.

For these reasons, adequate funding and payment mechanisms need to be provided to support the provision of molecular diagnostic tests such as companion diagnostics now and into the future.

\section{Challenges and Recommendations to Improve Reimbursement and Equitable Patient Access to Companion Diagnostics in France}

\section{Challenges}

In France, achieving patient access to new companion diagnostic tests is a long and difficult process.

Depending on the setting where the new test will be conducted, reimbursement will require a global payment code (i.e. GHS or BHN/AHC) for the public hospital/laboratory scenario, and a fee schedule code (i.e. NABM/ CCAM) for the private hospitals/laboratories.

Based on budgetary processes, public hospitals/laboratories can readily purchase and uptake CE-marked new tests. If private laboratories want to be reimbursed for performing those tests (even for hospitalised patients), a new NABM/CCAM tariff is required. To achieve this, a request needs to be made to the Medical Devices $\mathrm{Na}$ tional Review Board - CNEDiMTS. Specifically, the CNEDiMTS board within the National Health Authority (HAS) will conduct the companion diagnostic reimbursement review, looking for evidence of clinical benefit. If this is considered positive, the dossier is forwarded
Wurcel et al. 
to the National Association of Health Insurances UNCAM - for pricing. The complete chain for performing the test is priced, and not the specific test. The request for review can only be made by specific bodies such as UNCAM, medical societies, or even be self-assigned by the HAS. Once the reimbursement and pricing process is complete, the diagnostic is listed on the national list for reimbursement code implementation, and hospitals and private commercial laboratories are able to make purchasing decisions.

HAS-UNCAM processes would benefit from more clarity on different steps and mechanisms to achieve reimbursement. The overall process may last several years, representing a barrier to the uptake of innovative diagnostics, especially in cases where a new NABM/CCAM code is required for tests conducted by private laboratories. During that time, access to standardised qualityassured tests will be limited, while non-standardised laboratory-developed tests would often be performed as an alternative. As an example, despite the plethora of CE-marked PCR tests, only a few PCR tests are listed in the NABM fee schedule, suggesting that the coding system is rarely updated for new molecular diagnostics tests [14].

In addition, although there are formally established steps for new code application, including health technology assessment processes based on evidence standards, in practice these processes are not necessarily followed, creating a disconnection between established guidelines and real decision making. As an example, in spite of the HAS favourable recommendations for molecular companion diagnostic testing, the UNCAM subsequent review for reimbursement and potential inscription on the national reimbursement lists can be pending for an additional and undefined period of time, such as in the cases of EGFR testing for lung cancer, BRAF testing for melanoma, KRAS and NRAS testing for colorectal cancer.

As previously mentioned, reviews conducted by the HAS can take more than a year, while CE-marked companion diagnostic tests wait for reimbursement and pricing. This represents a significant delay for patient access, in a particularly disadvantageous environment for manufacturers, where the intrinsic value of the companion diagnostic information is not recognised in reimbursement and funding decisions.

\section{Recommendations}

- Allow IVD manufacturers to request initiation of the reimbursement process for companion diagnostic tests.

Value of Companion Diagnostics
- Clarify and accelerate the companion diagnostic reimbursement process.

- Recognize and reward the intrinsic value of the companion diagnostic information in reimbursement and funding decisions.

- Recognize the value and differences between CEmarked tests and non-standarised tests in funding and reimbursement decisions.

\section{Common Challenges for Access to Companion Diagnostics across the EU}

Equal access to personalised health care is still an issue across the EU, as European countries may face different possibilities to handle health care expenditures. While this is a relevant issue to consider, challenges to access companion diagnostics may be sought beyond budget constraints, given the minimal fraction of the total health care expenditure they represent [15].

Health technologies such as companion diagnostics that can limit ineffective medication use, preventing unnecessary ambulatory consultations and hospitalisations, may enable to contain the overall health care costs, shifting resources to areas of need. This, in turn, could contribute to make personalised health care more affordable across the EU.

For example, a multi-gene recurrence score assay companion diagnostic test to select breast cancer patients who will not benefit from additional chemotherapy has demonstrated savings in the United States in terms of adjuvant chemotherapy, supportive care, and management of adverse events of USD 1,930 per patient tested (based on a $34 \%$ reduction in chemotherapy use) [16].

By using KRAS companion diagnostic testing to select metastatic colorectal cancer patients suitable for EGFR inhibitor therapy (cetuximab or panitumumab), USD 7,500-12,400 per patient could be saved in the United States and EUR 3,900-9,600 per patient could be saved in Germany with equivalent clinical outcomes. Using KRAS testing would save over EUR 115 million per year in Germany, considering a metastatic colorectal cancer incidence of 30,000 cases [17].

Challenges for equal access to companion diagnostics in Europe relate to the variation of approaches for assessment and reimbursement across and within member states, the general lack of link between assessments and decision making, and the lack of established clear processes for reimbursement, funding and uptake of diagnostic innovation.

Public Health Genomics 2016;19:137-143 141 
In the different EU countries, the reimbursement processes for innovative diagnostics are usually difficult, unclear, time consuming, and, in some countries, not able to be initiated by manufacturers. Companion diagnostics must undergo various and heterogeneous reimbursement and funding processes both across and within member states. In most EU nations, decisions to fund companion diagnostic tests are taken in parallel by various institutions within a decentralised funding approach. These local levels make access decisions based on different, lengthy and often unclear processes, procedures and requirements. This heterogeneity can impact the speed of adoption of companion diagnostic tests and provides uncertainty to tailor clinical and economic evidence. The length of these processes (years) represents another challenge manufacturers must face to achieve access within the health care systems. The reimbursement of a companion diagnostic may also depend on whether it is applied in the hospital or ambulatory setting, with the presence of silo budgets not stimulating the uptake even when there is a perceived need.

The differences in health technology assessment (HTA) systems, methods and requirements between and within countries, and the fact that they rarely inform reimbursement, funding or uptake decisions for companion diagnostics further complicates the picture. Manufacturers have no predictability or clarity regarding necessary steps for value demonstration and how this will lead to effective funding and uptake of the diagnostic test, lacking incentives to invest in evidence development.

As a result, coverage for many companion diagnostic tests varies across Europe. For example, while the oncology drug trastuzumab is widely reimbursed across the EU, reimbursement for its HER-2 companion diagnostic test varies across Europe. In the UK, France, Germany, and Italy, HER-2 testing is publicly funded, but in Spain, the pharmaceutical manufacturer funds the majority of testing, not assuring the continuation of this funding stream in the future.

Guidelines related to HTA methodology for companion diagnostics often do not account for the specificities of the diagnostic component, including methods very closely related to that used to assess medicines, posing unrealistic and non-fit-for-purpose evidence requirements which further impede value demonstration and patient access. They reflect the fact that assessments across Europe may not be fit for purpose for in vitro diagnostics, with different and non-specific methodologies being applied. Furthermore, assessments usually lack inclusion of decision maker's needs and values in the pro- cess, challenging their ultimate usefulness for decision making.

Reimbursement and funding for companion diagnostics do not recognise the value of information for patients and decision makers that the tests provide. This lack of recognition as to the inherent value of the information the diagnostic component brings to the 'joint product', diagnostic-treatment combination, is reflected in the fact that the reimbursement level is fixed based only on the value of the medicine, on historical use and/or competitive pricing.

Another key hurdle for access relates to the lack of clarity on financing streams for companion diagnostics and their sustainability over time. The case for the funding of BRAF mutation test in metastatic melanoma and anaplastic lymphoma, leading to a reliance on interim funding directly from the pharmaceutical companies developing the companion drug (UK) or through national agencies, such as the Institut National du Cancer (INCa) in France, is an example.

The uncertainty on access, funding and uptake of molecular diagnostics, such as companion diagnostics, has a direct influence on investment decisions. As higher uncertainty leads to lower access to external funding for the development of innovative molecular tests, innovation may be hampered. This is of particular concern for smalland medium-sized enterprises, which represent $95 \%$ of the European in vitro diagnostic industry, and are key drivers of diagnostic innovation in Europe.

\section{Recommendations to Ensure Patient Access to Companion Diagnostics and Build an Equitable and Affordable Personalised Health Care Strategy in Europe}

- Develop clear, transparent and timely market access processes for companion diagnostics in all European health systems, by establishing reimbursement processes, with associated specific funding streams and uptake mechanisms.

- Overcome silo budget structures, such as different funding streams for pharmaceuticals versus diagnostics, ambulatory versus inpatient care, among others.

- Improve the efficiency, clarity and speed of the existing reimbursement processes for diagnostic tests.

- Recognise and reward the value of diagnostic information in the assessment, reimbursement and funding decisions of diagnostic-medicine combinations.

- Avoid creating new barriers, such as non-fit-for-purpose HTA, which can limit access to valuable diagnostic innovation and personalised health care in Europe. 
- If HTA is applied to companion diagnostics, health systems need to clearly establish beforehand, how the assessment results will inform the pathways for uptake of the diagnostic innovation. Whenever value assessments are made, these should be linked to decisions on reimbursement and/or uptake.

- HTA processes should enable patient access to companion diagnostics, by improving the way they assess their value, performing the assessment at the right time in the technology's life cycle, and responding to the needs of patients and decision makers.

- HTA needs to develop and use specific and pragmatic methodologies for the assessment of companion diagnostics, being different than the ones applied for medicines and therapeutic technologies, in order to bring forward the full value of companion diagnostics.

\section{Conclusion}

For a successful and affordable personalised health care strategy that is able to reach all European patients who will benefit from it, challenges around effective mar- ket access to companion diagnostics need to be addressed by most member states. Europe needs to avoid creating new barriers to innovation such as non-fit-for-purpose HTA initiatives.

National health systems and European initiatives should ensure that an innovation-friendly environment exists, uptake of valuable diagnostic innovation is encouraged, and patient access to diagnostics is improved.

For those objectives to be reached, clear, transparent and timely market access processes based on the value of the information provided by the diagnostic test need to be in place.

With these critical aspects taken into account, together with continued collaboration among health systems, patients, health care professionals and in vitro diagnostic manufacturers, personalised health care can bring the future of health care to the present.

\section{Disclosure Statement}

The authors represent the interests of the previously mentioned in vitro diagnostic manufacturers associations, at both the European and national levels.

\section{References}

1 Henshall C, Schuller T; HTAi Policy Forum: Health technology assessment, value-based decision making, and innovation. Int J Technol Assess Health Care 2013;29:353-359.

2 National Human Genome Research Institute: All about the human genome project. https:// www.genome.gov/10001772.

3 German Social Code No. 5, Article 35a SGB V. http://www.ilo.org/dyn/natlex/natlex4. detail?p_lang=en\&p_isn $=41168$.

4 German Social Code No. 5, Article 87 para. 3e SGB V. https://www.bundestag.de/blob/ $284870 /$ ce 0 d 03414872 b427e $57 \mathrm{fccb}$ 703634dcd/basic_law-data.pdf.

5 Concentra: Molecular diagnostic provision in England for targeted cancer medicines (solid tumour) in the NHS. A report for Cancer Research UK. 2015. http://www.cancerresearchuk.org/sites/default/files/policy_ august2015_mdx_final.pdf.

6 Department of Health: Improving outcomes: a strategy for cancer. 2011. https://www.gov. uk/government/uploads/system/uploads/ attachment_data/file/213785/dh_123394.pdf.

7 Department of Health: Ensuring equitable access to complex molecular diagnostic testing for cancer patients. 2012. http://ukgtn.nhs.
uk/fileadmin/uploads/ukgtn/Documents/ Resources/Library/Consultations/ UKGTN\%20response $\% 20$ to\%20Cancer\%20 Molecular\%20Tests\%20\%20submitted\%20 19.06.12.pdf.

8 Department of Health: Ensuring equitable access to complex molecular diagnostic testing for cancer patients. 2012. http://www.bivda. co.uk/News/PolicyandMedia/tabid/63/articleType/ArticleView/articleId/765/Default.aspx.

9 The British In Vitro Diagnostics Association (BIVDA): Molecular diagnostic testing - a submission to the Cancer Task Force. 2015. http://www.bivda.co.uk/.

10 Cancer Research UK: UK government missing the target over new cancer drug tests. 2014. http://scienceblog.cancerresearchuk. org/2014/12/01/uk-government-missingthe-target-over-new-cancer-drug-tests/.

11 Cancer Taskforce: Achieving world-class cancer outcomes: a strategy for England 20152020. 2015. http://www.cancerresearchuk. org/sites/default/files/achieving_worldclass_cancer_outcomes_-_a_strategy_for_ england_2015-2020.pdf.

12 The British In Vitro Diagnostics Association (BIVDA): Robust, fair and sustainable: our vision for the future access and provision of molecular diagnostics in England. 2015. http://www.bivda.co.uk/.

13 NHS England: Improving outcomes: a strategy for cancer - fourth annual report. 2014. https://www.gov.uk/government/uploads/ system/uploads/attachment_data/ file/388160/fourth_annual_report.pdf.

14 L'Assurance Maladie: Codage des actes biologiques - NABM. http://www.ameli.fr/professionnels-de-sante/medecins/exercer-auquotidien/nomenclatures-et-codage/codagedes-actes-biologiques-nabm.php.

15 MedTech Europe: The European Medical Technology industry in figures. Analysis from WHO, Eurostat, EFPIA, EDMA, Eucomed calculations. http://www.medtecheurope. org/node/282.

16 Michaela A, et al: Association between use of the 21-gene recurrence score assay and receipt of chemotherapy among Medicare beneficiaries with early-stage breast cancer, 2005-2009. JAMA Oncol 2015;1:1098-1109.

17 Vijayaraghavan A: Cost-effectiveness of KRAS testing in metastatic colorectal cancer patients in the United States and Germany. Int J Cancer 2012;131:438-445. 